\title{
Crosstalk between ATF4 and MTA1/HDAC1 promotes osteosarcoma progression
}

\author{
Heng Zeng ${ }^{1, *}$, Jin-ming Zhang ${ }^{1, *}$, Yu Du ${ }^{1,2, *}$, Jiang Wang ${ }^{1}$, Ye Ren ${ }^{1}$, Mi Li ${ }^{1}$, Hao Li ${ }^{1}$, \\ Zhuo Cai ${ }^{1}$, Qian $\mathrm{Chu}^{3}$, Caihong Yang ${ }^{1}$ \\ ${ }^{1}$ Department of Orthopedics, Tongji Hospital, Huazhong University of Science and Technology, Wuhan, Hubei 430030, P.R. China \\ ${ }^{2}$ Department of Orthopedic Surgery, The Second Affiliated Hospital, Chongqing Medical University, Chongqing 400016, \\ P.R. China \\ ${ }^{3}$ Department of Oncology, Tongji Hospital of Huazhong University of Science and Technology, Wuhan, Hubei 430030, P.R. China \\ * These authors have contributed equally to this work
}

Correspondence to: Caihong Yang, e-mail: yangcaihong1688@163.com

Keywords: activating transcription factor 4, metastasis-associated protein 1, osteosarcoma, histone deacetylase 1

Received: July 31,2015 Accepted: January 01, 2016 Published: January 18, 2016

\section{ABSTRACT}

The stress response gene activating transcription factor 4 (ATF4) is involved in metastatic behavior and cellular protection. Here we show that ATF4 is upregulated in osteosarcoma (OS) cell lines and patient clinical samples as compared to matched non-tumor tissue. Overexpression of ATF4 in OS cells promoted cell proliferation, migration and lung metastasis. Furthermore, the expression of ATF4 was markedly reduced in metastasis associated protein (MTA1) or histone deacetylase 1 (HDAC1) knockdown OS cells, but MTA1 overexpression increased the stability and activity of ATF4 protein via ATF4 deacetylation by HDAC1. ATF4 in turn enhanced the expression of MTA1 and HDAC1 at the transcription level, suggesting a positive feedback loop between ATF4 and MTA1/HDAC1. Clinically, the level of ATF4 was positively correlated with that of MTA1 in OS. Mice injected with ATF4-overexpressing cells exhibited a higher rate of tumor growth, and the average weight of these tumors was $90 \%$ greater than the controls. Taken together, these data establish a direct correlation between ATF4-induced OS progression and MTA1/HDAC1-associated metastasis, and support the potential therapeutic value of targeting ATF4 in the treatment of OS.

\section{INTRODUCTION}

Osteosarcoma (OS) is a highly malignant bone tumor in children and adolescents [1]. In $15-30 \%$ of OS cases, pulmonary metastases are observed at initial diagnosis [2]. Although several signaling pathways have been found to be involved in OS pathogenesis, the molecular mechanisms underlying OS progression have not yet been fully elucidated. It is necessary to identify new targets to enhance the efficacy of OS treatment and improve long-term survival of OS patients.

Activating transcription factor 4 (ATF4) belongs to the ATF/cyclic AMP response element binding protein (CREB) family [3]. ATF4 expression is increased in response to stresses in the tumor microenvironment [4]. Paradoxically, ATF4 was shown to activate both proliferation and apoptosis. For example, ATF4 increased VEGF-dependent tumor growth triggered by osteopontin, whereas excessive ATF4 activation was separately shown to promote tumor cell death $[5,6]$. Despite being considered a global regulator of osteoblast biology and bone metabolism [7], the role of ATF4 in OS is poorly understood.

Metastasis-associated protein (MTA), including MTA1, MTA2, and MTA3, is a component of the nucleosome remodeling and histone deacetylation (NuRD) complex [8]. MTA1 expression and its clinicopathologic relevance have been widely investigated in various cancers, including OS $[9,10]$. MTA1 functions as a transcriptional coactivator or corepressor of various genes. In most human cancers, overexpression of MTA1 promotes disease progression [11]. However, crosstalk between ATF4 and MTA1 remains largely unstudied.

The present study was aimed at exploring the function of ATF4 in OS. Our results revealed that MTA1/ HDAC1 promoted stabilization of ATF4 protein. ATF4 
increased MTA1 and HDAC1 expression, suggesting a positive feedback loop between ATF4 and MTA1/HDAC1. In addition, ATF4 showed pro-tumor activity in vitro and in vivo, suggesting the potential therapeutic value of targeting ATF4 in the treatment of OS.

\section{RESULTS}

\section{ATF4 overexpression promotes OS growth and metastasis}

Immunohistochemical staining showed that ATF4 expression was significantly higher in clinical OS tissue than in control samples (Figure 1A), suggesting that ATF4 plays an important role in OS development. Compared with the control, ATF4 overexpression promoted proliferation in U2OS cells (Figure 1B and 1C), and ATF4U2OS cells exhibited increased invasive potential to an extent greater than $80 \%$ (Figure 1D), and higher migratory ability (Figure 1E), when compared to vector-U2OS cells.

To examine ATF4 in vivo effects, control or ATF4U2OS cells were subcutaneously injected into the right flanks of mice and tumor growth was evaluated. Figure $1 \mathrm{~F}$ indicates that all mice injected with ATF4-U2OS cells exhibited a higher rate of tumor growth compared with control cells, and the average weight of ATF4-U2OS tumors was $\sim 90 \%$ more than that of control tumors. ATF4U2OS-injected mice also exhibited earlier tumor growth than controls.

As shown in Figure 1G (left panel), all mice in the ATF4 group had gross evidence of pulmonary metastatic lesions, whereas no lung nodules were visible in the control group, which was further confirmed with H\&E staining (middle panel). The average number of pulmonary nodules in the ATF4 group was 9.2, as compared to 0.2 in the control group (right panel). Additionally, ATF4 knockdown inhibited OS growth and metastasis (data not shown).

\section{MTA1 physically interacts with ATF4}

As both MTA1 and ATF4 proteins are expressed in malignant OS [12], we investigated the possibility of crosstalk between MTA1 and ATF4. To this end, we first examined whether the two proteins could interact. Even though HEK293 cells have endogenous expression of MTA1 or ATF4 (Figure 2A), Myc-MTA1 coimmunoprecipitates Flag-ATF4 in HEK293 cells (Figure 2B, left panel). We also observed this binding in a reverse experiment (Figure $2 \mathrm{~B}$, right panel). We also found evidence of binding between the endogenous MTA1 and ATF4 in 143B and ZOS cells (Figure 2C). To analyze the region of ATF4 required for binding to MTA1, we constructed several deletion mutants based on its reported domain structure (Figure 2D), and applied them in an in vitro GST pull-down assay. The result demonstrated that both the p300 interaction site (1-90) and the central region including ODDD and $\beta$-TrCP recognition motifs (140-230) of ATF4, corresponded to the MTA1 binding domain. However, the C-terminal domain (270-351) was not required for the interaction with MTA1 (Figure 2D). Thus, recombinant MTA1 directly binds with the $\mathrm{N}$ terminus of ATF4 (amino acids 1-224).

\section{MTA1 stabilizes ATF4 protein by inhibiting its ubiquitination}

Next, using MTA1+/+ or MTA1-/- MEF cell lines [13], we examined the role of MTA1 depletion on the levels of endogenous ATF4. Compared with ATF4 levels in wildtype MEFs, a marked reduction in the level of ATF4 protein in the MTA1-/- MEFs was observed (Figure 3A). However, this ATF4 reduction could be reverted by reintroduction of MTA1 in the MTA1-/- MEFs (Figure 3B). We found no discernible differences in ATF4 mRNA levels among the MTA1+/+, MTA1-/-, and MTA1-/-/MTA1 MEFs (Figure $3 \mathrm{C})$, suggesting a post-translational role of MTA1 in increasing the level of ATF4 protein. Furthermore, MTA1 knockdown in the 143B cells induced a reduction in ATF4 protein (Figure 3D), whereas overexpression of MTA1 in the 143B cells increased the steady-state levels of ATF4 (Figure 3E). Treatment with cycloheximide to inhibit protein synthesis further indicated that ATF4 stability increased in cells cotransfected with MTA1, but decreased in MTA1-depleted cells (data not shown).

ATF4 stability is determined through a series of post-translational modifications, including ubiquitination, which primarily relies on a phosphorylation-dependent interaction with the SCF ( $\beta$-TrCP) ubiquitin ligase [14]. To test whether MTA1 stabilizes ATF4 by inhibiting its ubiquitination, we examined the role of MTA1 on endogenous ATF4 ubiquitination in 143B-MTA1 stable cells. As shown in Figure 3F, overexpression of MTA1 decreased endogenous ATF4 ubiquitination and increased ATF4 protein. Similarly, Myc-MTA1 expression in HEK293 cells also suppressed ATF4 ubiquitination (Figure 3G). These findings suggest that MTA1 stabilizes ATF4 protein at least in part by inhibiting its ubiquitination.

We also measured the transcriptional activity of ATF4 using the luciferase reporter vector containing six tandem copies of ATF4 binding site (6 x ARE) [15]. Cotransfection of the MTA1 vector into U2OS cells activated the reporter activity, even in the absence of stress (Figure 3H).

\section{MTA1 promotes binding of ATF4 with HDAC1, which leads to ATF4 deacetylation}

It was shown that MTA1 increased the stabilization of HIF- $1 \alpha$ and p53 proteins by deacetylation, which is critically determined by HDAC1 recruitment [16]. Therefore, we examined whether HDAC1 was involved in the MTA1induced stabilization of ATF4. As shown in Figure 4A, the 

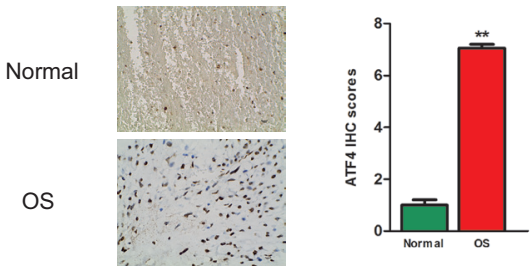

C

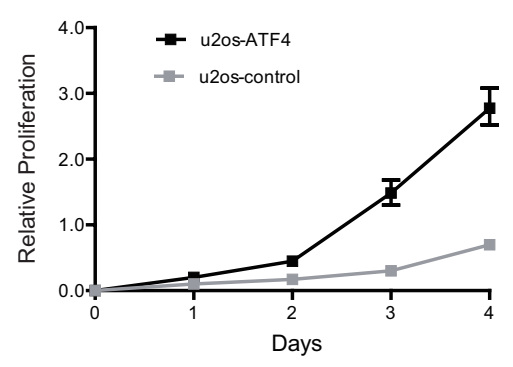

E
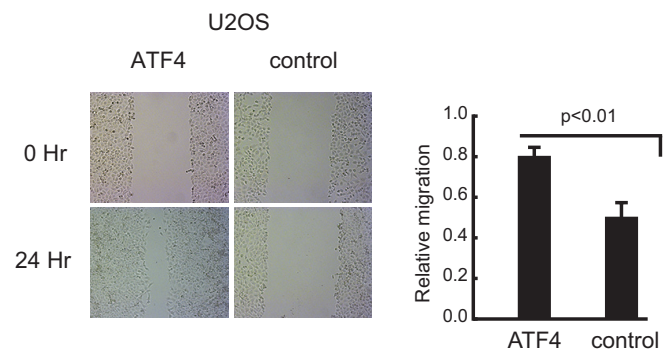

G
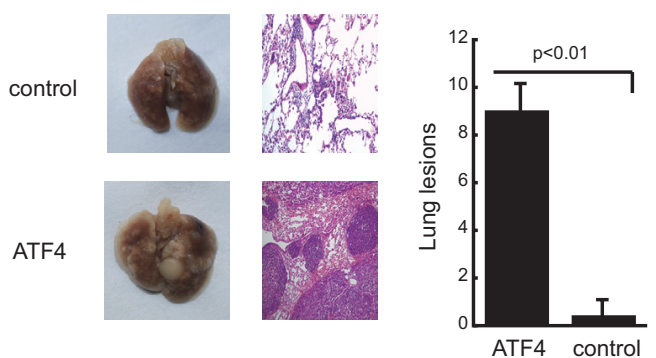

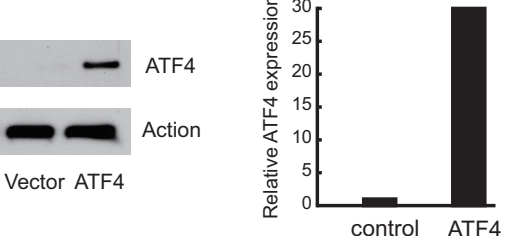

D

ATF4
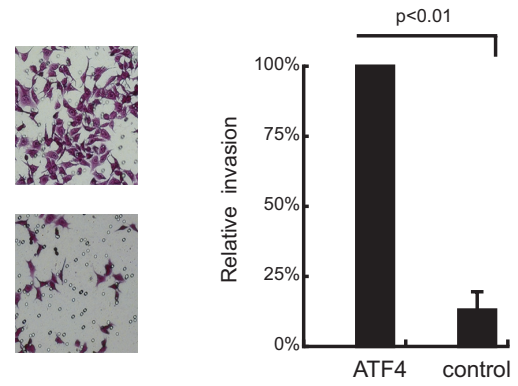

F

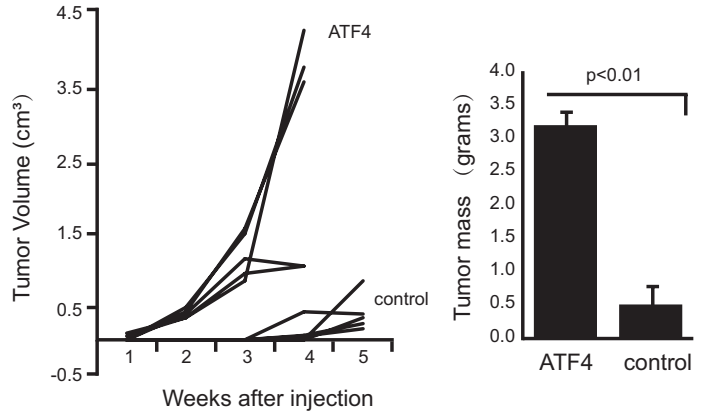

Weeks after injection 
A

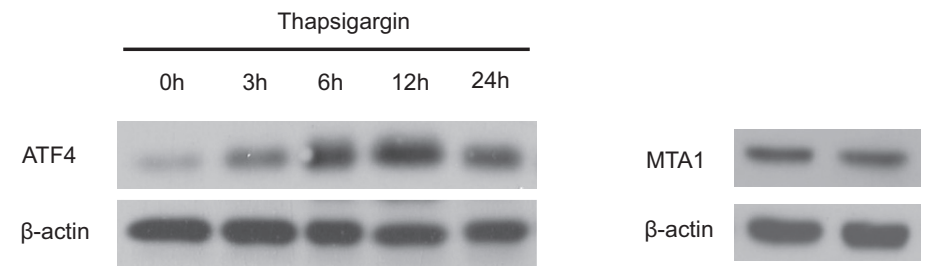

B

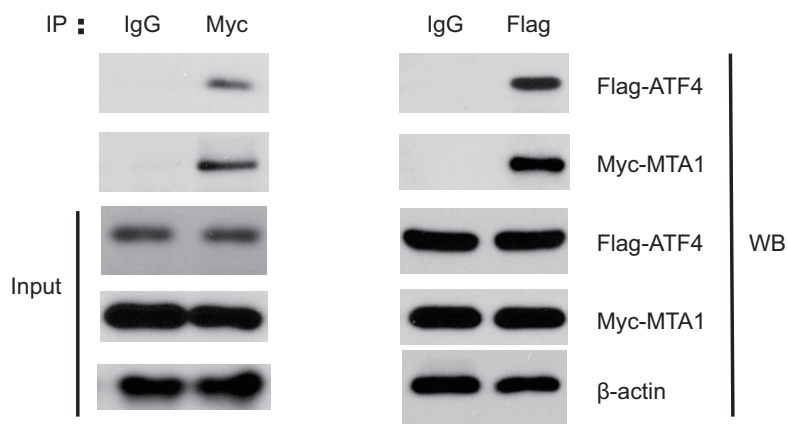

C
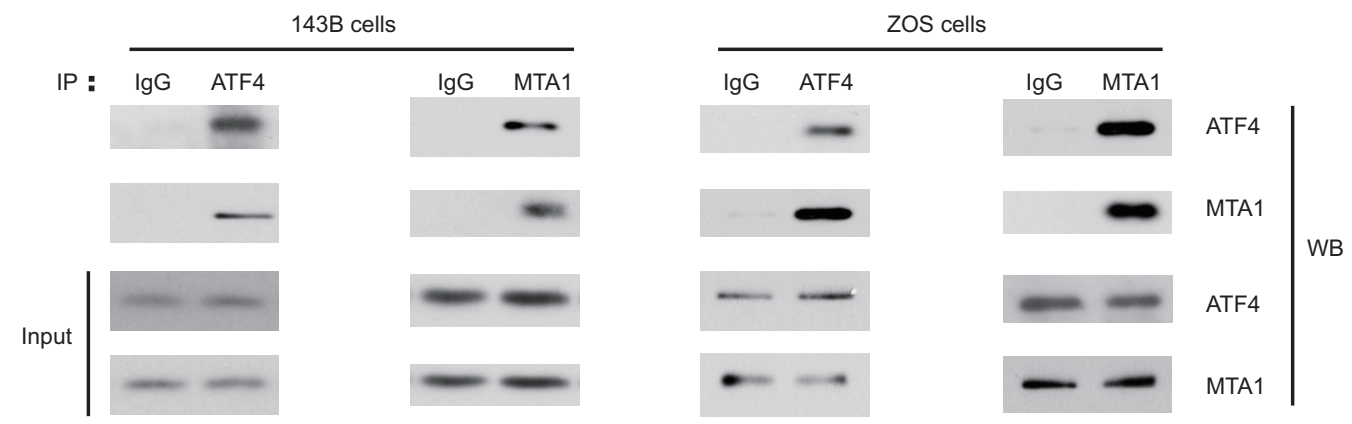

D

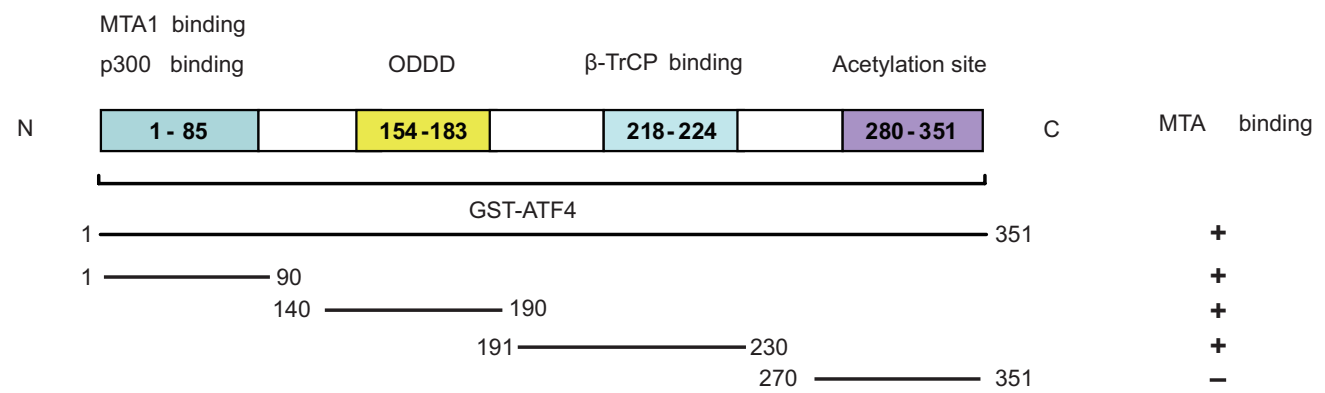

Figure 2: MTA1 physically binds with ATF4. Changes in ATF4 under ER stress induced by Thapsigargin (Tg) A. HEK293T cells were treated with $\operatorname{Tg}(10 \mathrm{nM})$ over increasing time periods $(0,3,6,12,24$ hours $)$. Cell lysates were immunoblotted using antibodies directed against ATF4 and $\beta$-actin. Endogenous MTA1 in HEK293 cells without treatment was also probed. B. HEK293 cells were transfected with expression vectors encoding Myc-MTA1 and Flag-ATF4 and immunoprecipitated with IgG control or specific antibodies against Myc or Flag, followed by immunoblotting with the indicated antibodies C. Protein extracts from 143B and ZOS cells were subjected to IP with antiATF4 or anti-MTA1 antibody or IgG control, followed by western blot analyses with the indicated antibodies D. Diagrammatic summary of in vitro-translated MTA1 binding with a series of GST-ATF4 proteins. 


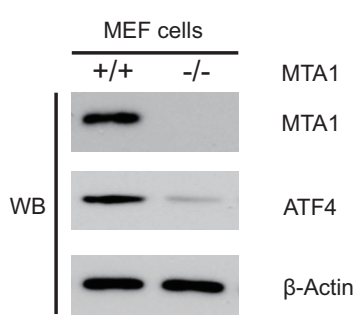

D

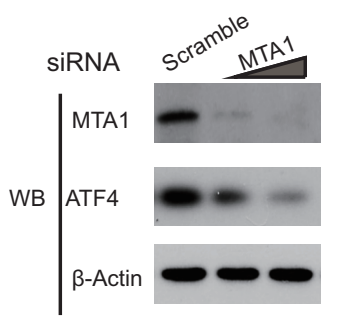

G

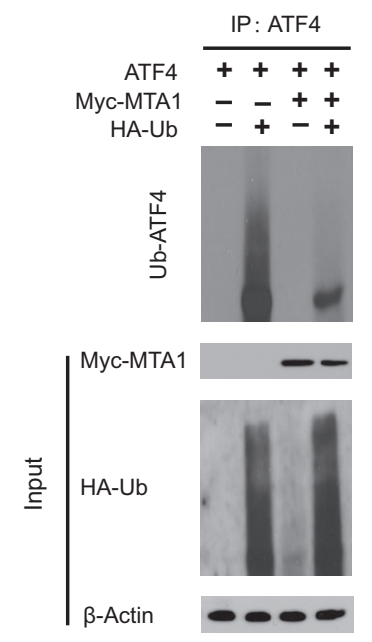

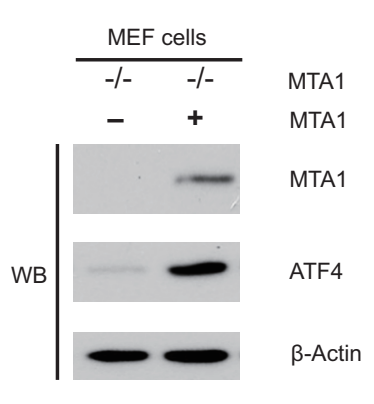

E

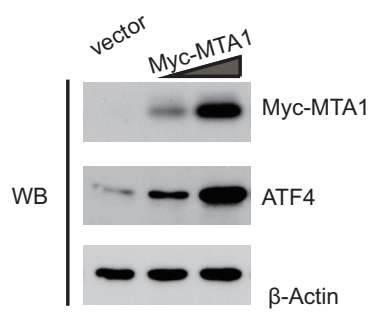

$\mathrm{H}$

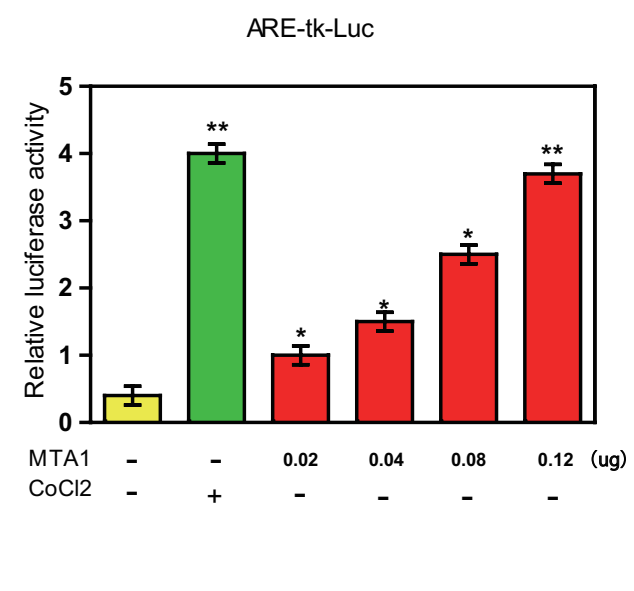

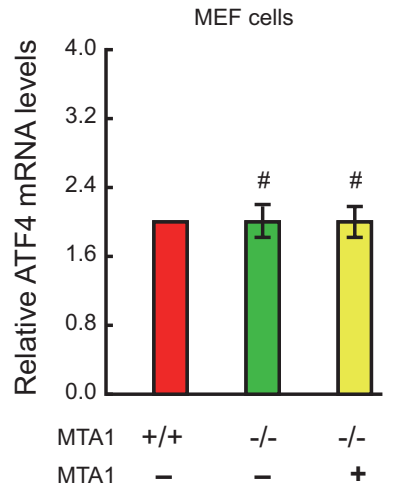

F

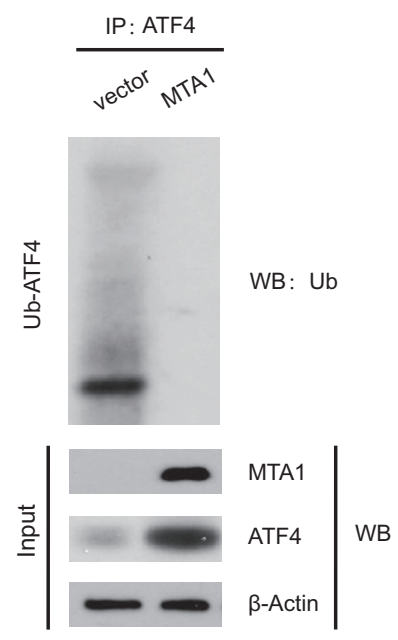

Figure 3: MTA1 stabilizes ATF4 protein and enhances its transcriptional activity. Protein extracts from indicated cell lines were subjected to western blot analysis with the indicated antibodies A and B. qPCR analysis of ATF4 mRNA levels in the indicated MEFs C. Western blot analysis of MTA1 and ATF4 protein expression in 143B cells transfected with increasing amounts of specific siRNA targeting human MTA1 or Myc-MTA1 expression plasmids D and E. Protein extracts from indicated cells were subjected to IP with an anti-ATF4 antibody, followed by western blot analysis with the indicated antibodies F. HEK293 cells were transfected with the indicated plasmids and subjected to IP and western blot analysis as described above G. The ARE-tk-Luc $(0.1 \mu \mathrm{g})$ reporter was cotransfected with the indicated amount of eukaryotic expression vector for MTA1 into U2OS cells H. Transfected cells were incubated for 24 hours in the presence or absence of $100 \mu \mathrm{M} \mathrm{CoCl}_{2}$, and luciferase activity was measured. Values are expressed as relative luciferase activity normalized to cotransfected renilla luciferase activity. 
expression of HDAC1 in 143B cells was largely induced under Thapsigargin (Tg)-treated ER stress, or via the overexpression of MTA1. Since there is a possibility that HDAC1 counteracts the acetylation of ATF4 and inhibits its degradation, we examined whether trichostatin A (TSA), a potent specific inhibitor of HDAC, decreased the stability of ATF4. As expected, TSA decreased the amount of ATF4 that was induced by either Thapsigargin or MTA1 expression, indicating that $\mathrm{HDACl}$ activity is closely correlated with the stability of ATF4.

A

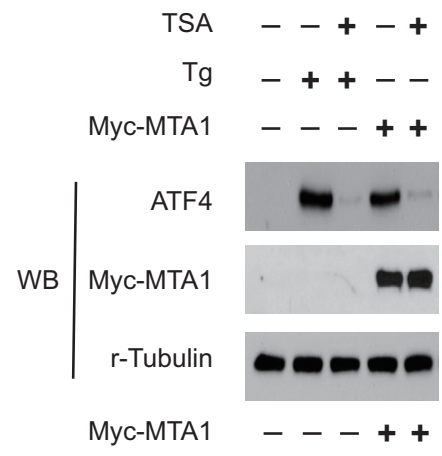

Next, we examined the relative amounts of acetylated ATF4 in the presence of MTA1. When cells were treated with MG132, a proteosome inhibitor, ATF4 acetylation increased significantly, but acetylated ATF4 was not detected in the presence of MTA1 or Thapsigargin. Similarly, compared with MG132 alone, treatment with MG132 and Thapsigargin or MTA1 significantly decreased the amount of acetylated ATF4. However, TSA treatment significantly increased ATF4 acetylation in the presence of MTA1 and MG132, suggesting that HDAC1

C

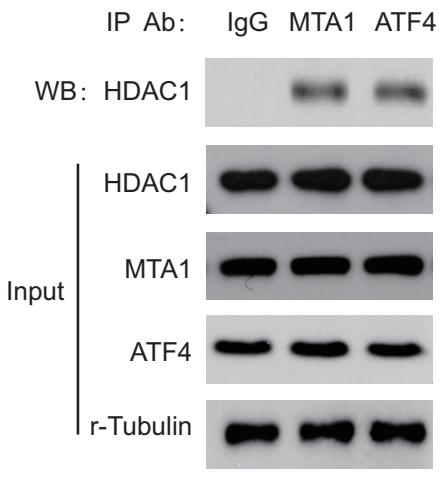

B

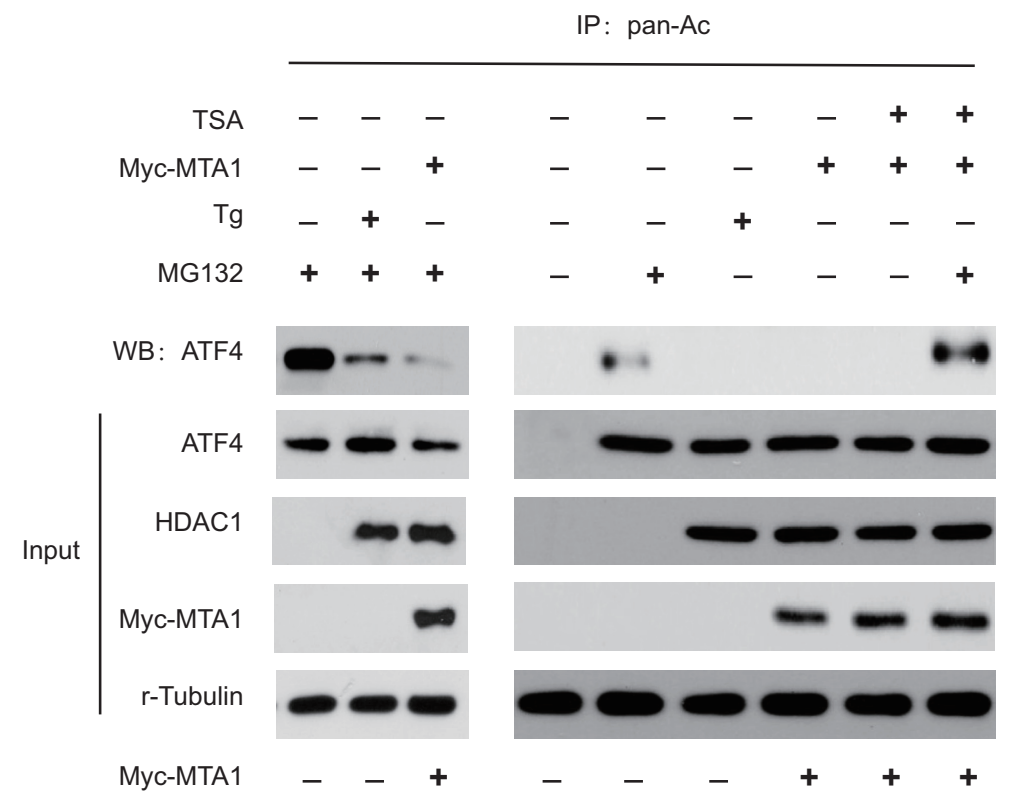

Figure 4: HDAC1 promotes the MTA1-induced stabilization of ATF4. 143B cells were transfected with Myc-MTA1 or empty vector A. Transfected cells were treated with or without thapsigargin (Tg) as indicated, then treated with or without $300 \mathrm{ng} / \mathrm{ml} \mathrm{TSA}$ for 3 hours. Cells were collected and lysed with RIPA buffer. The expression of ATF4, HDAC1, MTA1, Myc-MTA1, and r-tubulin in whole lysates was analyzed by western blot (WB) analysis. 143B cells were treated with $10 \mu \mathrm{M}$ MG132 or $300 \mathrm{ng} / \mathrm{ml}$ TSA for 1 or 3 hours, respectively B. Cell lysates ( $700 \mu \mathrm{g}$ ) were immunoprecipitated (IP) with anti-pan-Ac antibody, and then probed using anti-ATF4 antibody. The expression of indicated proteins was analyzed by western blot analysis. 143B cells were treated with Tg for 24 hours C. Cell lysates were immunoprecipitated with normal anti-IgG, anti-MTA1, or anti-ATF4 antibodies, and then probed using anti-HDAC1 antibody. The expression of ATF4, HDAC1, MTA1, and r-tubulin in the input was analyzed by western blot analysis. 
deacetylates ATF4 and thereby blocks ATF4 degradation (Figure 4B).

Given that MTA1 protein binds physically to HDAC1 [16], we examined the association between ATF4, MTA1, and HDAC1 in the presence of Thapsigargin. As shown in Figure 4C, MTA1, HDAC1 and ATF4 were efficiently co-precipitated, indicating that these proteins are capable of forming a complex in vivo in the presence of Thapsigargin. Together, our data indicate that MTA1 inhibits the acetylation of ATF4 by recruiting HDAC1, and increases the stabilization of ATF4 in OS, suggesting that HDAC inhibitors could potentially be used to treat OS.

\section{MTA1 dictates the binding of ATF4 to PHD1, PHD3 and $\beta$-TrCP in OS cells}

ATF4 protein abundance is dictated by two prolyl hydroxylase domain-containing proteins, PHD1 and PHD3, in mammalian cells, but not by PHD2 [17]. These PHDs belong to the superfamily of oxygenases that require $\mathrm{O}_{2}, 2$-oxoglutarate (2-OG), and $\mathrm{Fe}^{2+}$ for their enzymatic activity [17]. The central region of ATF4 including the ODD domain and $\beta$-TrCP recognition motif is involved in binding to PHD1. The F-box protein $\beta$-TrCP, the receptor component of the SCF E3 ubiquitin ligase, is colocalized in the nucleus with ATF4 and decreases its stability [14]. Because MTA1, HDAC1, PHD1, PHD3 and $\beta$-TrCP bind to ATF4 protein and control its stability [14], we investigated whether MTA1 decreases the binding of ATF4 to each of these. In OS cells, both PHD3 and $\beta$-TrCP, but not PHD1, strongly bound to ATF4 in the presence of MG132, suggesting that binding of PHD3 and $\beta$-TrCP to ATF4 precedes ATF4 degradation. However, in the presence of MTA1, binding of ATF4 to PHD3 or $\beta$-TrCP was largely decreased, whereas binding between ATF4 and PHD1 increased (Figure 5A). Similar results were observed when the ODD domain of ATF4 was used (Figure 5B). Moreover, MTA1 knockdown in OS cells significantly reversed acetylation and binding of ATF4 (Figure 5C), indicating that MTA1 predominantly blocks ATF4 ubiquitination via ATF4 deacetylation.

\section{ATF4 transcriptionally activates the expression of MTA1 and HDAC1 in OS}

Given that ATF4 is a transcriptional factor, we studied whether ATF4 enhances expression of MTA1 and HDAC1 in OS cell lines in which expression of the ATF4 gene is under control of an inducible doxycycline promoter. Expression levels of MTA1 and HDAC1 proteins were dramatically increased with doxycycline treatment (Figure 6A). Moreover, the mRNA levels of MTA1 and HDAC1 were increased, indicating that ATF4 induces the expression of MTA1 and HDAC1 genes at the transcription level (Figure 6B).
Analysis of the human MTA1 and HDAC1 promoters revealed potential binding sites for ATF4, and ChIP assays detected stress-inducible binding of ATF4 to these sites (Figure 6C). siRNAs against ATF4 significantly reduced MTA1 and HDAC1 transcription after ER stress (Figure 6D).

Next, we examined whether ATF4 could increase MTA1/HDAC1 activity. To this end, we carried out deacetylation assays using purified GST-MTA1, FlagHDAC1, histone $\mathrm{H} 4$ and $[3 \mathrm{H}]$-acetyl CoA. Whereas histone H4 was acetylated as expected, recombinant ATF4 significantly decreased H4 acetylation (Figure 6E), indicating that ATF4 promoted MTA1 activity.

We also found that ATF4 expression dramatically decreased the amount of ubiquitinated MTA1 protein (Figure 6F). Together, these data reveal an unexpected feedback loop among ATF4, MTA1 and HDAC1, which is likely to have significant consequences in environmentally compromised OS tissues and cells.

\section{Correlation between ATF4 and MTA1 abundance}

We found a significant positive correlation between the abundance of ATF4 and MTA1 in nine OS cell lines and in a cohort of OS patients (Figure 7A and 7B). Together, these results strongly support the hypothesis that the observed crosstalk between ATF4 and MTA1 has physiological importance in OS.

\section{DISCUSSION}

In this study, we show that ATF4 promotes OS growth and metastasis. MTA1 stabilizes ATF4 by recruiting $\mathrm{HDAC} 1$, and ATF4 transcriptionally activates the expression of MTA1 in OS. Two mechanisms that could account for ATF4 protein stability have been described: (a) SCF $\beta$-TrCP degrades ATF4 and (b) histone acetyltransferase p300 increases ATF4 stabilization by inhibiting ATF4/ $\beta$-TrCP binding and subsequent degradation [14, 18, 19]. Although p300 can acetylate ATF4, its role in ATF4 stabilization is not due to p300 catalytic activity. p300 binds to the N-terminal region of ATF4, leading to sequestration of ATF4 into the nuclear speckles, where it is unavailable for $\beta$-TrCP binding [18]. This provides insight into a new mechanism for stabilization of ATF4 by MTA1, in which enzymatic activity of HDAC1 and deacetylation of ATF4 are required, and MTA1 inhibits binding of ATF4 with $\beta$-TrCP. Phosphorylation by an unknown kinase is probably required for subsequent recognition by SCF $\beta$-TrCP [14]. Our data suggest that HDAC1 binds to ATF4 and promotes ATF4 stability via deacetyltransferase activity. Hence, we propose a molecular basis for the cell specificity of ATF4 accumulation in OS. 


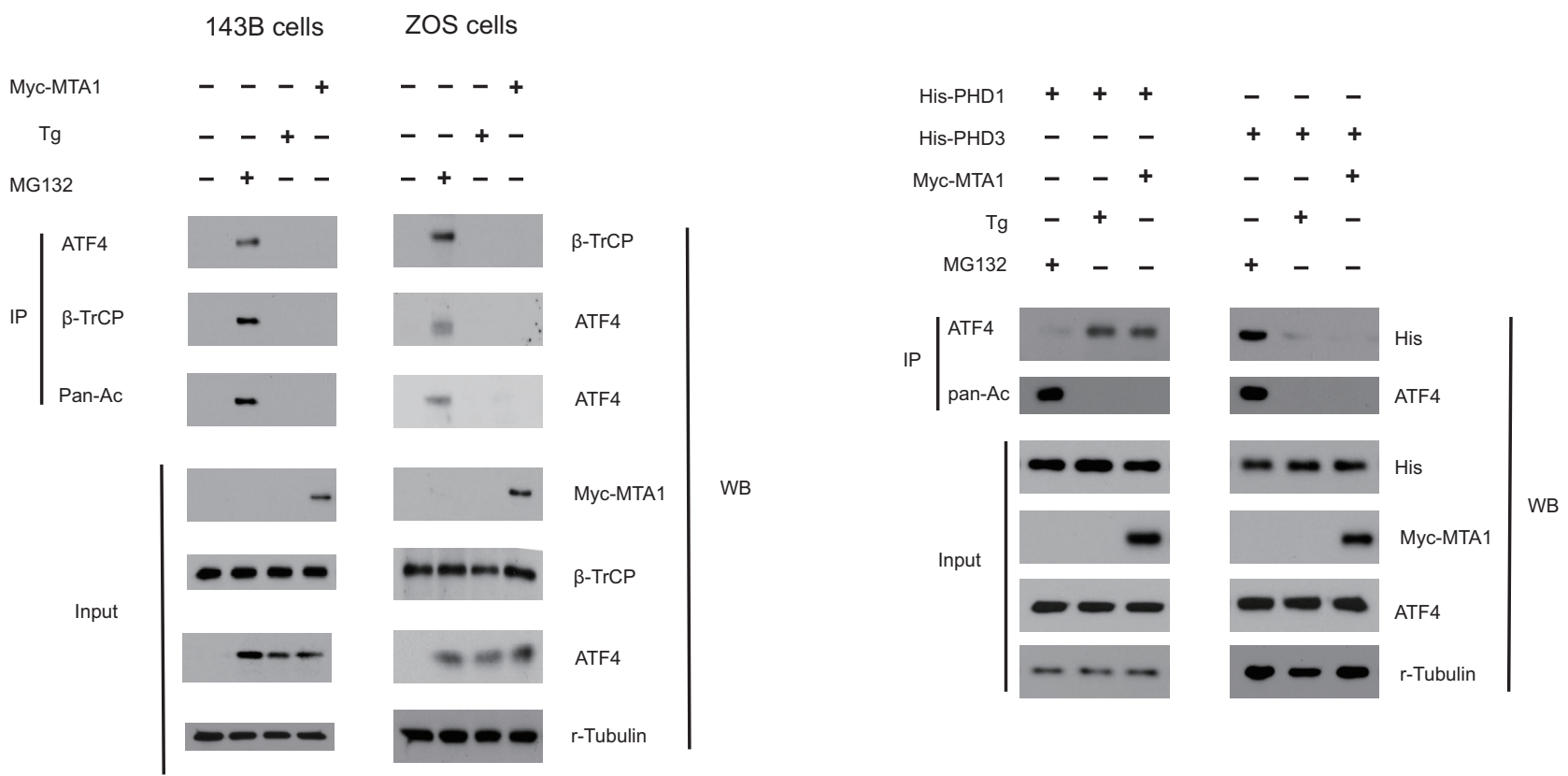

C

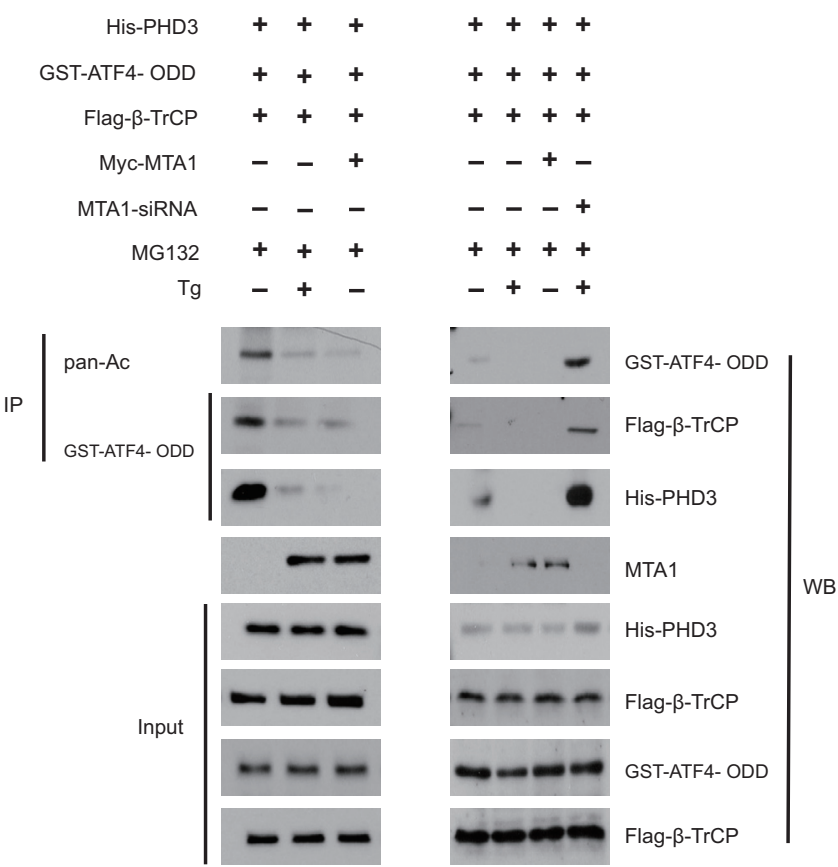

Figure 5: MTA1 dictates the binding of ATF4 to PHD1, PHD3 and $\boldsymbol{\beta}$-TrCP in OS cells. Myc-MTA1 or empty vector were transfected into 143B and ZOS cells A. Transfected cells were treated without (NT) or with thapsigargin (Tg) or $10 \mu \mathrm{M}$ MG132 for 1 hour before being harvested as indicated. Cell lysates were immunoprecipitated (IP) and then probed with indicated antibodies. 143B cells were transfected with His-PHD1 or His-PHD3, with or without Myc-MTA1 as indicated B. Transfected cells were treated as described in (A). IP and western blot were completed with indicated antibodies. 143B cells were transfected with GST-ATF4- ODD (aa 140-190), Flag- $\beta$-TrCP, His-PHD1 and His-PHD3 with Myc-MTA1 or MTA1-siRNA C. Cells were treated with or without $10 \mu$ M MG132, and IP and western blotting were performed. 


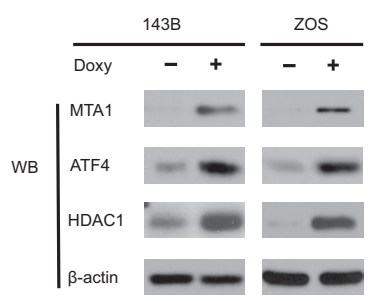

C
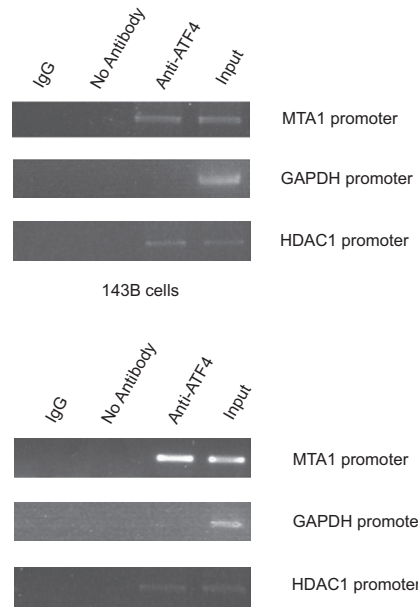

MTA1 promoter

GAPDH promoter HDAC1 promoter ZOS cells
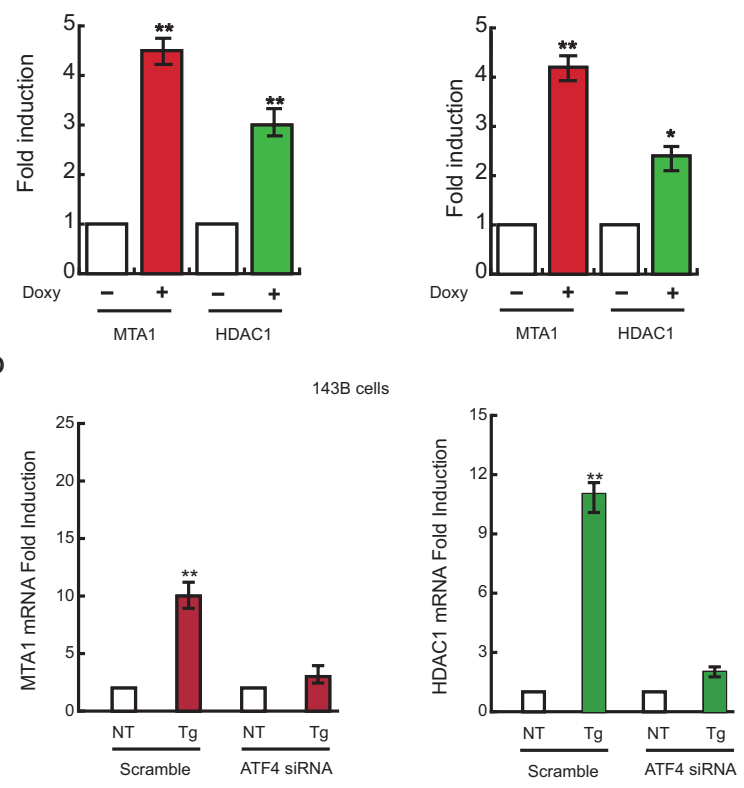

zos cells
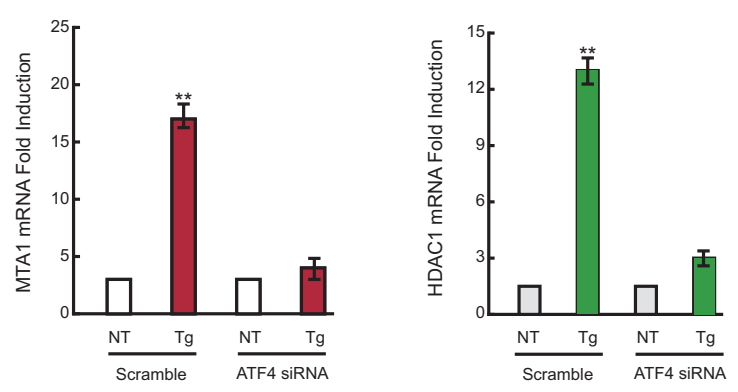

$\mathrm{F}$
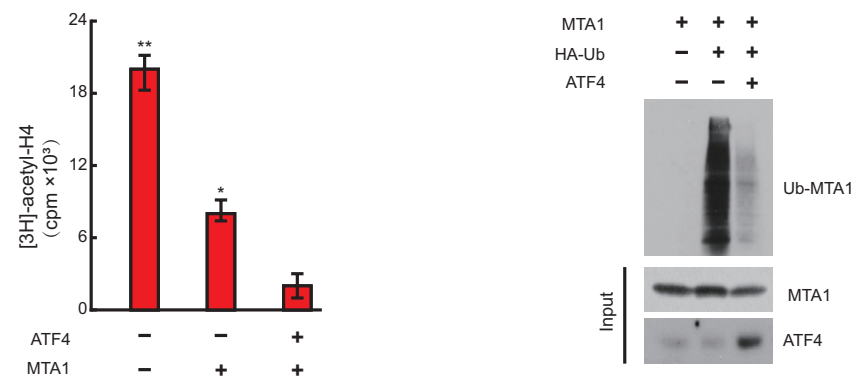

Figure 6: ATF4 induces the expression of MTA1 and HDAC1 at the transcription level. 143B and ZOS cells were treated with vehicle or $2 \mu \mathrm{g} / \mathrm{ml}$ of doxycycline (doxy) for 24 hours A. Proteins were analysed by western blot analysis. 143B and ZOS cells were transfected and treated as described in panel A, and gene expression was analyzed by quantitative real-time PCR B. ChIP assay showing the binding of ATF4 to MTA1 and HDAC1 promoter in vivo $\mathbf{C}$. The promoter regions of MTA1 and HDAC1 were amplified from the DNA recovered from the immunoprecipitation complex using a specific antibody for ATF4. The input DNA, ChIP yield using nonspecific IgG and GAPDH promoter are included as controls. 143B and ZOS cells were transiently cotransfected with either siRNA specific for human ATF4 or a non-targeting negative control siRNA (Scramble) D. After 48 hours, cells from each group were either left untreated (NT) or treated with thapsigargin (Tg). Cells were harvested after 8 hours, total RNA was extracted, and MTA1 and HDAC1 mRNA levels were determined following the indicated stress conditions. MTA1 activity was determined by incubating $0.6 \mu \mathrm{g}$ of Myc-MTA1 and Flag-HDAC1 with $5 \mu \mathrm{l}$ of $\left[{ }^{3} \mathrm{H}\right]$-acetyl CoA, $0.6 \mu \mathrm{g}$ of acetylated histone H4, p300 and/or $1 \mu \mathrm{g}$ of recombinant ATF4 E. Data are presented as mean \pm SEM $(n=3)$. ATF4 expression decreased MTA1 ubiquitination F. Lysates from 143B cells transfected with $1 \mu \mathrm{g}$ of Myc-MTA1, HA-ubiquitin and/ or ATF4 were subjected to immunoprecipitation using the Myc antibody followed by SDS-PAGE. Ubiquitinated proteins were detected by western blotting using the HA antibody. 
ATF4 was shown to induce expression of several target genes, including asparagine synthesase, Gadd153, VEGF, TRB3, osteocalcin, TFIIB and RAP30 [20, 21, 22]. Additionally, ATF4 transactivated gene expression by binding to various partners, including $\mathrm{Nrf} 2$, to induce heme oxygenase-1 (HO-1), or through its deacetylation by HDAC1 $[23,24]$. We demonstrated that MTA1 and HDAC1 were induced by ATF4 in OS, suggesting a positive feedback loop between ATF4 and MTA1/HDAC1.

Given that ATF4 is upregulated by signals in the tumor microenvironment, such as hypoxia [12], and is closely correlated to angiogenesis and adaptation of cancer cells to hypoxia [25], inhibiting ATF4 might be especially attractive for treating OS patients. Kai et al reported a novel pathway of chromatin remodeling by blocking the MTA1/HDAC1 complex, and suggested a combined treatment of resveratrol, which decreased MTA1 expression, and the HDAC inhibitor, SAHA [26]. Although this treatment cooperatively increased p53 levels and apoptosis in prostate cancer, it was unclear whether ATF4 expression was changed. In sum, our finding indicate that the ATF4/MTA1/HDAC1 axis promotes the development of OS. Therefore, inhibitors of HDAC1, ATF4 and MTA1 may prevent OS carcinogenesis.

\section{MATERIALS AND METHODS}

\section{Cell culture, antibodies and reagents}

U2OS and 143B human osteosarcoma cell lines were originally purchased from ATCC, and cultured in DMEM and RPMI 1640 medium supplemented with $10 \%$ fetal bovine serum, respectively. ZOS cells were established from the primary tumor of an osteosarcoma patient and described previously [13, 27]. Human osteosarcoma 143B cells (ATCC ${ }^{\circledR}$ CRL-8303 ${ }^{\mathrm{TM}}$ ), are suitable transfection host cells, thymidine kinase deficient (TK-) and resistant to BUdR [28]. Transfections were carried out using Lipofectamine 2000 (Invitrogen) according to the manufacturer's instruction. All reagents in this study were of analytical grade and are commercially available. All mouse embryonic fibroblasts (MEFs) cell lines used in this study are from Dr. Rakesh Kumar's laboratory and have been described [13]. To establish cell lines stably expressing MTA1, MTA1-/- MEFs were transfected with expression vector encoding pcDNA6/V5MTA1 or empty vector using FuGENEHD Transfection Reagent (Roche Applied Science, Indianapolis, IN). After 24 hours of transfection, cells were subjected to selection with $10 \mu \mathrm{g} / \mathrm{ml}$ blasticidin (Invitrogen) for 2 weeks. The resulting colonies were isolated and analyzed for V5MTA1 expression by immunoblotting.

The primary antibodies used in this study were purchased from the following companies: the gammatubulin and $\beta$-actin antibodies were from Sigma, Germany. anti-MTA1, anti-ATF4 (sc-22800), Anti-HA (sc-805) and anti-Myc (9E10) antibodies were obtained from Santa Cruz Biotechnology. Anti-HA (MMS-101P) antibody was obtained from Covance. Anti-HDAC1 (10E2) antibody was obtained from Abcam. Anti- GAPDH was obtained from Millipore. The alkaline phosphatase-conjugated anti-rabbit, anti-mouse or anti-goat IgGs or anti-FLAG were also purchased from Sigma. The FITC- and Cy3conjugated IgGs were purchased from the PTG lab, USA. DAPI (4, 6-diamidino-2-phenylindole dihydrochloride, ( $2 \mu \mathrm{g} / \mathrm{ml}$ methanol)) was purchased from Taufkirchen, Germany.

\section{RNA extraction, semiquantitative RT-PCR and real-time $q P C R$}

Total RNA extraction was performed using an RNeasy kit (Qiagen). RNA concentration and purity were measured using a NanoDrop spectrophotometer (Thermo Scientific). RNA was retro-transcribed using SuperScript III reverse transcriptase (Life Technologies) for use in subsequent PCR reactions. Platinum TAQ (Life Technologies) was employed for semi-quantitative RT-PCR. Real time qPCR was performed using TaqMan universal master mix II (Life Technologies) and different
A

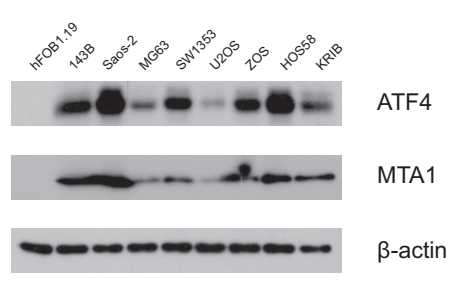

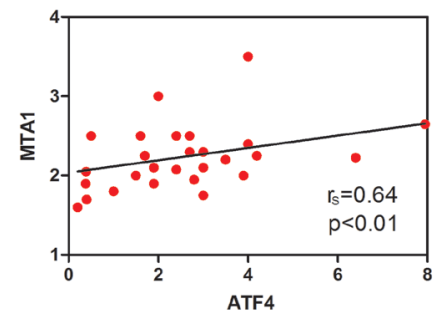

B

\begin{tabular}{|c|c|c|c|c|c|}
\hline MTA1 $^{\text {ATFA }}$ & 0 & + & ++ & +++ & Total \\
\hline 0 & 7 & 3 & 2 & 2 & 14 \\
\hline+ & 6 & 4 & 13 & 7 & 30 \\
\hline++ & 1 & 1 & 10 & 11 & 23 \\
\hline+++ & 0 & 1 & 2 & 45 & 48 \\
\hline Total & 14 & 9 & 27 & 65 & 115 \\
\hline
\end{tabular}

Figure 7: The association between ATF4 and MTA1. ATF4 abundance was positively associated with that of MTA1 (P < 0.01 ) in a panel of human OS cell lines A. Correlation analyses were performed with the Spearman rank correlation test. ATF4 abundance was associated with MTA1 in 113 primary human OS specimens B. Correlation analyses were performed with the Pearson chi-square test. 
TaqMan assays. Primers used for RT-PCR and qPCR were: ATF4 (F): 5'-ATGGCCGGCTATGGATGAT-3' and (R): 5'- CGAAGTCAAACTCTTTCAGATCCATT-3'; GAPDH (F): 5'-GAAATCCCATCACCATCTTCCAGG-3' and (R): 5'- CGCGGCCATCACGCCACAGTTTCC-3'; MTA1 (F): 5'- GCAGCTGAAGCTGAGAGCAAGTTA-3' and (R): 5'-CCTTGACGTTGTTGACGCTGA-3'. $\quad \beta$-actin (F):5'-CGTGGGCCGCCCTAGGCACCA-3', and (R): 5'-TTGGCTTAGGGTTCAGGGGGG-3'.

\section{Ubiquitination assays}

For ubiquitination assays, indicated cells transfected with FLAG-ATF4 or myc-MTA1 and HA-ubiquitin were treated with $5 \mu \mathrm{M}$ of MG132 overnight, and then lysed in the FLAG lysis buffer (50 mM Tris-HCl, pH 7.9, 137 $\mathrm{mM} \mathrm{NaCl}, 10 \mathrm{mM} \mathrm{NaF}, 1 \mathrm{mM}$ EDTA, 1\% Triton X-100, $0.2 \%$ sarkosyl and $10 \%$ glycerol). Cell lysates (1-2 mg) were incubated with $20 \mu \mathrm{l}$ of anti-FLAG M2 affinity gel (Sigma) at $4^{\circ} \mathrm{C}$ overnight. After extensive washes, agarose gels were loaded on spin columns (Affymetrix), and bound FLAG-ATF4 or MTA1 was eluted with $20 \mu \mathrm{l}$ of FLAG peptide at a final concentration of $0.1 \mathrm{mg} / \mathrm{ml}$. ATF4 or MTA1 ubiquitination was determined by western blotting.

\section{Cell viability assay}

OS cells were cultured overnight in a 96-well plate at a density of $5 \times 10^{3}$ cells/well. Control cells or cells stably overexpressing ATF4 were cultured in the presence of 5\% FBS for 48 hours. Cell viability was assessed by MTT assay (Thermo Scientific, USA). The number of surviving cells was assessed by measuring absorbance at $490 \mathrm{~nm}$ for the dissolved formazan product after addition of $20 \mu \mathrm{L}$ MTT solution $(0.5 \mathrm{mg} / \mathrm{ml})$ for 4 hours. Cell counting kit- 8 (CCK-8; Dojindo, Japan) was used to determine OS cell proliferation based on the manufacturer's protocol. Briefly, cells were seeded in 96-well plates at a density of $5 \times 10^{3}$ cells/well and were treated as MTT assay. At different time points, $10 \mu \mathrm{L}$ of CCK- 8 solution was added to each well and incubated for 1 hour at $37^{\circ} \mathrm{C}$. Absorbance (optical density, OD) was measured at $450 \mathrm{~nm}$.

\section{Wound healing and transwell invasion assay}

For migration assay, cells were seeded in 6-well plates and grown to $80 \%$ confluency. The cell monolayers were scraped off with a sterile plastic tip and were cultured in DMEM with $1 \%$ FBS under normoxia $\left(20 \% \mathrm{O}_{2}\right)$ or hypoxia $\left(1 \% \mathrm{O}_{2}\right)$. Pictures were taken under a microscope at 0 and 24 hours points, and the distance between the two boundaries of an acellular area was measured. The results of the different treatment groups were expressed as a ratio to the distance of 0 hour point. In vitro invasive activity through a gel matrix was examined in 24-well plates. Cells were resuspended at a density of $1 \times 10^{5}$ cells/ ml. BD Falcon Cell Culture Inserts (San Jose, CA, USA) were coated with $50 \mu \mathrm{L}$ of Matrigel and placed in each well. Wells were filled with $600 \mu \mathrm{L}$ of complete medium, and the upper inserts were filled with $100 \mu \mathrm{L}$ of the cell suspension. After 24 hours, viable invasive cells that adhered to the lower surface of the filter were fixed using $70 \%$ methanol and stained with $0.5 \%$ crystal violet. Cells on the upper surface of the filter were removed with cotton swabs. The cells in the lower level were counted.

\section{Establishment of an in vivo lung metastasis model}

Male BALB/c nude mice aged 3 to 4 weeks were purchased from Beijing Medical Experimental Animal Center (Beijing, China). All animal protocols were approved by the Institutional Animal Research Committee of Tongji Medical College. Control cells or cells stably overexpressing ATF4 or shATF4 were suspended in PBS and then subcutaneous and intramuscular injected into one nude mouse. Mice were sacrificed and the tumors were isolated. Tumor volume was calculated as (length $\times$ width $\left.^{2}\right) / 2$. Volumes were calculated twice a week. Control tumors were harvested on days 34 and 40, and tumor weights were measured. Lung samples were harvested for further examination, fixed with formalin and embedded in paraffin. The number of lung metastases was determined by counting the number of metastatic nodules on the lung surface. H\&E staining was performed to detect the lung metastases.

\section{Chromatin immunoprecipitation (ChIP)}

OS cells were washed twice with PBS and crosslinked with $1 \%$ formaldehyde for 10 minutes at room temperature (RT). Formaldehyde was quenched with 0.2 $\mathrm{M}$ glycine for 10 minutes at RT. Cells were then lysed for 10 minutes with rotation at $4^{\circ} \mathrm{C}$ in $1 \mathrm{ml}$ buffer $\mathrm{LB} 1$ (50 mM Hepes-KOH pH7.5, 140 mM NaCl, 1 mM EDTA, $10 \%$ glycerol, $0.5 \%$ NP-40 and $0.25 \%$ Triton X-100). Nuclei were pelleted and washed first with $1 \mathrm{ml}$ buffer LB2 (10 mM Tris-HCl pH8.0, $200 \mathrm{mM} \mathrm{NaCl,} 1$ mM EDTA $\mathrm{pH} 8.0$ and $0.5 \mathrm{mM}$ EGTA pH 8.0) and then washed and resuspended in $750 \mu \mathrm{l}$ buffer LB3 $(10 \mathrm{mM}$ Tris- $\mathrm{HCl} \mathrm{pH} 8.0$, $200 \mathrm{mM} \mathrm{NaCl}, 1 \mathrm{mM}$ EDTA pH 8.0, 0.5 mM EGTA pH 8.0, $0.1 \%$ Na-deoxycholate, $0.5 \%$ N-lauroylsarcosine and protease inhibitors). Lysates were sonicated and centrifuged. After removing $5 \%$ of the supernatant volume (input), the remainder was split into two aliquots and incubated overnight with $30 \mu \mathrm{l}$ protein $\mathrm{G}$ magnetic beads coated with ATF4 or control IgG antibody. The beads were then resuspended in buffer WB I (50 mM Tris- $\mathrm{HCl} \mathrm{pH} 8.0$, $150 \mathrm{mM} \mathrm{NaCl}, 0.1 \%$ SDS, $0.1 \%$ Na-deoxycholate, $1 \%$ Triton X-100, 1 mM EDTA). They were then washed once with WB I, once with WB II (50 mM Tris- $\mathrm{HCl} \mathrm{pH} 8.0$, $500 \mathrm{mM} \mathrm{NaCl}, 0.1 \% \mathrm{SDS}, 0.1 \% \mathrm{Na}$-deoxycholate, $1 \%$ 
Triton X-100, $1 \mathrm{mM}$ EDTA), once with WB III (10mM Tris- $\mathrm{HCl}$ pH8.0, $250 \mathrm{mM} \mathrm{LiCl,} \mathrm{0.5 \%} \mathrm{NP-40,} \mathrm{0.5 \%} \mathrm{Na-}$ deoxycholate, $1 \mathrm{mM}$ EDTA) and twice with TE buffer. Beads were eluted with $200 \mu 10$ mM Tris- $\mathrm{HCl} \mathrm{pH} 8.0$, $0.5 \% \mathrm{SDS}, 300 \mathrm{mM} \mathrm{NaCl}$ and $5 \mathrm{mM}$ EDTA with shaking at $65^{\circ} \mathrm{C}$ for 30 minutes, and then were reverse crosslinked at $65^{\circ} \mathrm{C}$ overnight together with the input. RNA and proteins were removed by digestion with RNase at $37^{\circ} \mathrm{C}$ for 30 minutes and with proteinase $\mathrm{K}$ at $55^{\circ} \mathrm{C}$ for 1 hour. Finally, the DNA was purified using QIAquick columns (Qiagen) and resuspended in $40 \mu \mathrm{l}$ water. ChIP DNA was detected by qPCR using SYBR (Life Technologies) and specific primers for MTA1 genomic promoter regions: (sense), 5'-AAAGAGCACGGCCGCTCCTGGA-3' and (anti-sense), 5'-GGTCCTCCGGCATTCCTCCCTGA-3'; control: (sense), 5'-ATCGCGCCTCCATTTTCC-3' and (anti-sense), 5'-ATTTTGGGTTGGGGTGAG-3'.

\section{Immunohistochemistry}

Dewaxed and rehydrated tissue sections were incubated overnight at $4^{\circ} \mathrm{C}$ with indicated antibodies and then washed with PBS. Biotinylated goat anti-rabbit IgG was then added to the sections for 30 minutes at RT. After the sections were washed with PBS, peroxidaseconjugated avidin was applied. Peroxidase activity was detected by exposing the sections to a solution of $0.05 \%$ 3, 3-diaminobenzidine and $0.01 \% \mathrm{H}_{2} \mathrm{O}_{2}$ in Tris- $\mathrm{HCl}$ buffer (3, 3-diaminobenzidine solution) for 3 to 6 minutes at RT. The sections were counterstained with hematoxylin. Images were scanned and analyzed using an Aperio Imagescope (Leica Biosystems). Immunohistochemical staining for MTA1/ATF4 in tissue was scored by two of the authors as positive or negative. An estimation of the immunohistochemical expression of the markers was determined by counting $\geq 100$ cells in random highpower fields. The frequency of positive cells for each antibody was reported semi-quantitatively as follows: $(-)$, No reaction; $(+)$, mild with $<30 \%$ of positive cells; $(++)$, moderate with $30-60 \%$ of positive cells; and $(+++)$, marked with $>60 \%$ of positive cells. A positive expression was defined as the staining of $>30 \%$ of the cancer cells $(++$ or +++$)$.

\section{Immunoprecipitation and western blot analysis}

Protein extracts were obtained from tissues or cells lysed in buffer containing Complete Protease Inhibitor Cocktail (Roche Applied Science, Germany). For immunoprecipitation assays, cells were lysed using the Pierce Co-IP kit (Thermo Scientific, USA) according to the manufacturer's instructions. Proteins were resolved by $10 \%$ SDS-PAGE, transferred to PVDF membranes (Millipore, USA), incubated with the appropriate primary and secondary antibodies and detected using the
ImmunoCruz Western Blotting Luminol Reagent (Santa Cruz, USA).

\section{Plasmids}

The eukaryotic expression vectors for the constructs, pEGFP-C7-MTA1 (GFP-MTA1), pEGFP-N1PHD1 (GFP-PHD1), pEGFP-N1-PHD2 (GFP-PHD2), pEGFP-N1-PHD3 (GFP-PHD3) were from Addgenes. The full-length MTA1 constructs were constructed by inserting corresponding PCR-amplified fragments into the EcoRI/XhoI sites of pCMV-Myc (Clontech). All of the new constructs were verified by DNA sequencing. The siRNA duplexes targeting MTA1 included siMTA1\#1 (5'-CCCUGUCAGUCUGCUAUAATT-3' and 5'-UUAUAGCAGACUGACAGGGTT-3') and siMTA1\#2 (5'-AAGACCCUGCUGGCAGAUAAATT-3' and 5'-UUUAUCUGCCAGCAGGGUCU UTT-3'). The control was nonspecific siRNA. The transfection of siRNAs was performed twice at 24-hour intervals with Oligofectamine ${ }^{\mathrm{TM}}$ reagent (Invitrogen) according to the manufacturer's protocol.

\section{Luciferase assay}

Reporter vectors containing six tandem copies of ATF4 binding site (6 x ARE) were co-transfected with a SV40-Renilla luciferase construct into U2OS cells using Oligofectamine ${ }^{\mathrm{TM}}$ reagent (Invitrogen). Two days after transfection, cells were lysed, and luciferase activity was determined using specific substrates in a luminometer according to the manufacturer's protocol. Transfection efficiency was normalized by determining the activity of Renilla luciferase.

\section{Clinical tissue samples}

One hundred thirteen OS and paired adjacent noncancerous tissue specimens were collected between 2011 and 2014 from the Tongji Hospital, Huazhong University of Science \& Technology. The study was approved by the Committee for Ethical Review of Research in China, and the patients signed informed consent forms. All tissue samples were obtained from patients with OS. Samples were collected during surgery and were immediately snap frozen in liquid nitrogen until RNA and protein extraction.

\section{Statistics}

All graphs were generated and all statistical analyses (Student's two-tailed $t$ test and log-rank test) were performed with GraphPad Prism software (GraphPad Software Inc.). Data shown are the mean \pm SD of three independent experiments. ${ }^{*} p<0.05 ; * * p<0.001$. 


\section{GRANT SUPPORT}

This project was supported by the following grants: Wuhan science and technology project (2014010202010091), 2014 China Education Ministry Scientific Research Foundation for Returned Overseas Chinese Scholars, and Natural Science Foundation of Hubei Province, China (2013CFB099).

\section{CONFLICTS OF INTEREST}

The authors declare no conflicts of interest.

\section{REFERENCES}

1. Hawkins DS, Rajendran JG, Conrad EU 3rd, Bruckner JD, Eary JF. Evaluation of chemotherapy response in pediatric bone sarcomas by [F-18]-fluorodeoxy-D-glucose positron emission tomography. Cancer. 2002; 94:3277-3284.

2. Amankwah EK, Conley AP, Reed DR. Epidemiology and therapies for metastatic sarcoma. Clinical epidemiology. 2013; 5:147-162.

3. Wang C, Guo F. Effects of activating transcription factor 4 deficiency on carbohydrate and lipid metabolism in mammals. IUBMB life. 2012; 64:226-230.

4. Blais JD, Filipenko V, Bi M, Harding HP, Ron D, Koumenis C, Wouters BG, Bell JC. Activating transcription factor 4 is translationally regulated by hypoxic stress. Molecular and cellular biology. 2004; 24:7469-7482.

5. Chakraborty G, Jain S, Kundu GC. Osteopontin promotes vascular endothelial growth factor-dependent breast tumor growth and angiogenesis via autocrine and paracrine mechanisms. Cancer research. 2008; 68:152-161.

6. Kadowaki H, Nishitoh H. Signaling pathways from the endoplasmic reticulum and their roles in disease. Genes. 2013; 4:306-333.

7. Wang W, Lian N, Ma Y, Li L, Gallant RC, Elefteriou F, Yang X. Chondrocytic Atf4 regulates osteoblast differentiation and function via Ihh. Development. 2012; 139:601-611.

8. Marzook H, Deivendran S, Kumar R, Pillai MR. Role of MTA1 in head and neck cancers. Cancer metastasis reviews. 2014; 33:953-964.

9. Reddy SD, Pakala SB, Molli PR, Sahni N, Karanam NK, Mudvari P, Kumar R. Metastasis-associated protein 1/histone deacetylase 4-nucleosome remodeling and deacetylase complex regulates phosphatase and tensin homolog gene expression and function. The Journal of biological chemistry. 2012; 287:27843-27850.

10. Sen N, Gui B, Kumar R. Role of MTA1 in cancer progression and metastasis. Cancer metastasis reviews. 2014; 33:879-889.

11. Zhang SX, Sanders E, Wang JJ. Endoplasmic reticulum stress and inflammation: mechanisms and implications in diabetic retinopathy. Journal of ocular biology, diseases, and informatics. 2011; 4:51-61.

12. Wang RA. MTA1--a stress response protein: a master regulator of gene expression and cancer cell behavior. Cancer metastasis reviews. 2014; 33:1001-1009.

13. Manavathi B, Peng S, Rayala SK, Talukder AH, Wang MH, Wang RA, Balasenthil S, Agarwal N, Frishman LJ, Kumar R. Repression of Six3 by a corepressor regulates rhodopsin expression. Proc Natl Acad Sci U S A. 2007, 7;104:13128-33.

14. Lassot I, Ségéral E, Berlioz-Torrent C, Durand H, Groussin L, Hai T, Benarous R, Margottin-Goguet F.ATF4 degradation relies on a phosphorylation-dependent interaction with the SCF(betaTrCP) ubiquitin ligase. Mol Cell Biol. 2001, 21:2192-2202.

15. Takahata Y, Hinoi E, Takarada T, Nakamura Y, Ogawa $\mathrm{S}$, Yoneda Y. Positive regulation by gamma-aminobutyric acid B receptor subunit-1 of chondrogenesis through acceleration of nuclear translocation of activating transcription factor-4. The Journal of biological chemistry. 2012; 287:33293-33303.

16. Kaur E, Gupta S, Dutt S. Clinical implications of MTA proteins in human cancer. Cancer Metastasis Rev. 2014, 33:1017-24.

17. Hiwatashi Y, Kanno K, Takasaki C, Goryo K, Sato T, Torii S, Sogawa K, Yasumoto K. PHD1 interacts with ATF4 and negatively regulates its transcriptional activity without prolyl hydroxylation. Exp Cell Res. 2011, 10;317:2789-99.

18. Liu J, Wang H, Huang C, Qian H. Subcellular localization of MTA proteins in normal and cancer cells. Cancer metastasis reviews. 2014; 33:843-856.

19. Suzuki T, Osumi N, Wakamatsu Y. Stabilization of ATF4 protein is required for the regulation of epithelialmesenchymal transition of the avian neural crest. Developmental biology. 2010; 344:658-668.

20. Balasubramanian MN, Butterworth EA, Kilberg MS. Asparagine synthetase: regulation by cell stress and involvement in tumor biology. American journal of physiology Endocrinology and metabolism. 2013; 304:E789-799

21. Dey S, Savant S, Teske BF, Hatzoglou M, Calkhoven CF, Wek RC. Transcriptional repression of ATF4 gene by CCAAT/enhancer-binding protein beta (C/EBPbeta) differentially regulates integrated stress response. The Journal of biological chemistry. 2012; 287:21936-21949.

22. $\mathrm{Su}$ N, Kilberg MS. C/EBP homology protein (CHOP) interacts with activating transcription factor 4 (ATF4) and negatively regulates the stress-dependent induction of the asparagine synthetase gene. The Journal of biological chemistry. 2008; 283:35106-35117.

23. Komatsuzaki E, Kitamura T, Murayama I, Saigo Y, Ojima K, Akatsuka N, Iwabuchi J, Miyata S. Characterization of an activating transcription factor 4 gene containing a consensus phosphorylation site for PKA in the gonads of Xenopus embryos. Zoological science. 2010; 27:19-23. 
24. Dey S, Sayers CM, Verginadis, II, Lehman SL, Cheng Y, Cerniglia GJ, Tuttle SW, Feldman MD, Zhang PJ, Fuchs SY, Diehl JA, Koumenis C. ATF4-dependent induction of heme oxygenase 1 prevents anoikis and promotes metastasis. The Journal of clinical investigation. 2015; 125:2592-2608.

25. Pettersen EO, Ebbesen P, Gieling RG, Williams KJ, Dubois L, Lambin P, Ward C, Meehan J, Kunkler IH, Langdon SP, Ree AH, Flatmark K, Lyng H, Calzada MJ, Peso LD, Landazuri MO, et al. Targeting tumor hypoxia to prevent cancer metastasis. From biology, biosensing and technology to drug development: the METOXIA consortium. Journal of enzyme inhibition and medicinal chemistry. 2014:1-33.

26. Kai L, Samuel SK, Levenson AS. Resveratrol enhances p53 acetylation and apoptosis in prostate cancer by inhibiting
MTA1/NuRD complex. International journal of cancer Journal international du cancer. 2010; 126:1538-1548.

27. Zou CY, Wang J, Shen JN, Huang G, Jin S, Yin JQ, Guo QC, Li HM, Luo L, Zhang M, Zhang LJ.Establishment and characteristics of two syngeneic human osteosarcoma cell lines from primary tumor and skip metastases. Acta Pharmacol Sin. 2008; 29:325-32.

28. Mohseny AB, Machado I, Cai Y, Schaefer KL, Serra M, Hogendoorn PC, Llombart-Bosch A, Cleton-Jansen AM. Functional characterization of osteosarcoma cell lines provides representative models to study the human disease. Lab Invest. 2011, 91:1195-205. 